\title{
Gluten-sensitive Enteropathy
}

\author{
INFLUENCE OF HISTOCOMPATIBILITY TYPE
}

ON GLUTEN SENSITIVITY IN VITRO

\author{
Z. M. Falchuk, D. L. Nelson, A. J. Katz, J. E. Bernardin, D. D. Kasarda, \\ N. E. HAgue, and W. STrober, Departments of Medicine and Pediatrics \\ (Gastroenterology Division), Peter Bent Brigham Hospital \\ and Children's Hospital Medical Center, Harvard Medical School, Boston, \\ Massachusetts 02115; Immunophysiology Branch, National Cancer Institute, \\ Bethesda, Maryland 20205; Western Regional Research Center, \\ U. S. Department of Agriculture, Berkeley, California 94710
}

A B S T RACT We previously developed an in vitro organ culture system in which gluten exerts a toxic effect on intestinal mucosa of patients with active gluten-sensitive enteropathy. Gluten generally inhibits the epithelial cell maturation of intestinal biopsy specimens that otherwise occurs if the tissue is cultured for $24-48 \mathrm{~h}$ in a gluten-free medium. However, small intestinal mucosa from $15-20 \%$ of patients with proven gluten-sensitive enteropathy fails to manifest the expected gluten-induced damage in vitro. In the present study, we explored the relation between in vitro gluteninduced intestinal damage and the presence of HLA-B8. We determined whether the patients' histocompatibility type (HLA-B8 positive or negative) influenced the ability of gluten protein to inhibit epithelial cell maturation of cultured intestinal biopsy specimens from patients with gluten-sensitive enteropathy.

Intestinal biopsies from 21 of 24 patients with gluten-sensitive enteropathy and HLA-B8 showed gluteninduced damage in vitro. On the other hand, intestinal biopsies from only 4 of 16 patients with gluten-sensitive enteropathy but without HLA-B8 showed gluteninduced damage in vitro. The difference in the effect of gluten in vitro between these two groups was statistically significant $(P<0.01)$. The data show a dichotomy between gluten-induced tissue damage in vivo and in vitro in HLA-B8 negative patients, suggesting that

Presented in part at the Annual Meeting of the American Federation of Clinical Research, Atlantic City, N. J., 3 May 1975, and published in abstract form 1975 (Clin. Res. 23: 254A.).

Address reprint requests to Dr. Falchuk, Peter Bent Brigham Hospital, Boston, Mass.

Received for publication 4 May 1979 and in revised form 31 March 1980.
HLA-B8 is important for gluten to manifest a cytotoxic influence in organ culture.

\section{INTRODUCTION}

We previously established an in vitro model of glutensensitive enteropathy (GSE) ${ }^{1}$ using organ culture techniques (1). The model depends upon the fact that $(a)$ intestinal brush border alkaline phosphatase enzyme activity in small intestinal tissue obtained from patients with active GSE is low, reflecting altered mucosal architecture and impaired epithelial cell maturation; $(b)$ during organ culture of the intestinal tissue in a glutenfree medium, there is a dramatic increase in alkaline phosphatase enzyme activity accompanied by morphologic maturation of epithelial cells; and (c) during culture of the intestinal tissue in a gluten-containing medium, brush border alkaline phosphatase activity does not increase appreciably and cells do not show morphologic evidence of maturation. Thus, inhibition of brush border alkaline phosphatase increase in vitro is an indication of gluten toxicity in vitro, just as the characteristic mucosal lesion is an indication of gluten toxicity in vivo.

In prior experiments, in vitro gluten sensitivity did not always correlate with in vito gluten sensitivity (2). We noted that in studies of $15-20 \%$ of patients, regardless of the presence of gluten protein in culture, no inhibition of brush border alkaline phosphatase enzyme increase occurred. No adequate explanation exists for this observation.

In view of the known association of GSE with certain

${ }^{1}$ Abbreviations used in this paper: GSE, gluten-sensitive enteropathy; PT digest, peptic tryptic digest. 
histocompatibility antigens (HLA-B8, HLA-DW3), (3-6), we determined in the present study whether the deleterious effect of gluten in vitro is restricted by the patient's histocompatibility type. Accordingly, we examined small intestinal tissue from patients with GSE who were positive or negative for HLA-B8 and determined the susceptibility of the tissue in vitro to the cytotoxic effect of gluten protein. The data developed allow the conclusion that histocompatibility antigens are important to the process by which gluten causes epithelial cell cytotoxicity in GSE.

\section{METHODS}

Mucosal tissue from HLA-B8 positive and negative patients with GSE were compared as to their susceptibility to gluteninduced toxicity in vitro. Small intestine tissue obtained from disease and normal control individuals was also studied.

Gluten toxicity in vitro was determined by the previously described organ culture technique (1). To determine whether the magnitude of any given difference between alkaline phosphatase values in gluten-containing and gluten-free cultures was indicative of gluten-induced toxicity, a statistical function derived in a previous study from a population of $39 \mathrm{pa}-$ tients with a flat mucosal lesion was used. 26 patients had GSE and 13 did not (2). The function allowed elimination of any arbitrary decision regarding presence of in vitro gluteninduced toxicity in any individual case. To derive this function, patients and control individuals were studied prospectively by conventional clinical and laboratory techniques, as well as by organ culture. Alkaline phosphatase values of gluten-containing vs. gluten-free cultures for any given patient or control individual were then plotted on an $x$ and $y$ axis, respectively. It was found that two distinct populations of values were obtained that could be separated by a boundary function. Using discriminant function analysis (7), a mathematical definition of this boundary was derived: $Z=(0.012$ $\times$ alkaline phosphatase value without gluten $)-(0.0284 \times$ alkaline phosphatase value with gluten) +1.9975 . Positive $Z$ values obtained with this function (corresponding to values falling on one side of the boundary function) described most patients who had GSE, while negative values (corresponding to values falling on the other side of the boundary function) described most patients without GSE. More specifically, $88 \%$ of patients with GSE and 7\% of disease-control individuals fell into one population, defined by positive $\mathrm{Z}$ values, whereas $12 \%$ of patients with GSE, 93\% of disease-control individuals, and $100 \%$ of normal individuals fell into another population, defined by negative $Z$ values. The classification error rates were the same for the observed sample and the jackknifed procedure. (It must be emphasized that the $\mathrm{Z}$ function analysis objectively determines the presence or absence of gluten sensitivity in vitro and not of gluten sensitivity in vivo. When applied to a random population of patients with abnormal small intestinal mucosa, the analysis correctly predicts the presence or absence of gluten sensitivity in vivo in $88 \%$ of patients. In the present study, the $\mathrm{Z}$ value was not used to predict the in vivo diagnosis, but to assess the in vitro effect of gluten.)

Study group. 40 patients with GSE, aged 11 mo-52 yr were studied. At the time of biopsy, the patients were either newly diagnosed and, therefore, on no dietary gluten restriction, or had previously defined GSE and were prepared for study by ingestion of gluten for at least $7 \mathrm{~d}$ before the study. The initial diagnosis was firmly established in all patients by the presence of diarrhea or malabsorption, typical appearance of small intestinal histology with total or subtotal villus flattening, infiltration of the lamina propria with lymphocytes and plasma cells, and vacuolization of the epithelial cells. All patients had appropriate response to gluten withdrawal and gluten challenge with remission and exacerbation, respectively, of clinical and laboratory features. The control group consisted of $(a) 8$ normal individuals with no history of gastrointestinal disease and normal intestinal morphology, (b) 12 patients with diarrhea not due to GSE and normal small bowel morphology, and (c) 15 patients with diarrhea and abnormal small bowel morphology not due to GSE. The latter patients included three with eosinophilic gastroenteritis, two with tropical sprue, five with hypogammaglobulinemia, three with chronic diarrhea of infancy, and two with cow's milk allergy. All patients, including those in the control groups, were eating a gluten-containing diet at the time of study. All individuals under the age of 18 in this study were being evaluated for malabsorption and/or diarrhea and underwent intestinal biopsies as part of a standard assessment. Informed consent was obtained from all individuals studied or their parents. These studies were approved by the Human Studies Committee of the Peter Bent Brigham Hospital Division, Affiliated Hospitals Center, Inc., the Children's Hospital Medical Center, and the National Institutes of Health Clinical Center.

Biopsy technique. A multi-purpose suction biopsy instrument, positioned fluoroscopically at the ligament of Treitz, was used to obtain specimens of jejunal tissue (8). Three or four biopsy specimens were routinely obtained and a portion of at least one specimen was sent for histological examination.

Organ culture. Biopsies were transported to the laboratory in iced RPMI 1640 medium with $10 \%$ fetal calf serum, insulin, and antibiotics. Each biopsy was cut into six to eight pieces, resulting in as many as 30 specimens, which were then thoroughly mixed. Three of these randomized specimens were placed villus-side-up on stainless steel wire mesh grids suspended over culture medium in culture dishes, thus insuring representation on the culture plate from each biopsy. The dishes were placed into closed containers, gassed with $95 \%$ oxygen and $5 \%$ carbon dioxide mixture, and incubated for $24-48 \mathrm{~h}$ at $37^{\circ} \mathrm{C}(1)$. Where indicated, one of two gluten preparations was added to the culture medium (see below). Culture specimens were removed from the culture dish grids at 24-48 $\mathrm{h}$ and homogenized in $1 \mathrm{ml}$ of $0.9 \%$ saline. Samples were assayed immediately or stored at $-80^{\circ} \mathrm{C}$ until assayed. Tissue protein (9) and alkaline phosphatase activity (10) were determined. 1 IU of alkaline phosphatase specific activity represented $1 \mu \mathrm{mol}$ of paranitrophenol phosphate hydrolyzed per gram of tissue protein per minute.

Gluten preparation. Two different preparations of gluten were used. A peptic tryptic (PT) digest of gluten was prepared according to the method of Frazer et al. (Frazer fraction III) (11). After digestion, pepsin and trypsin were inactivated by heating at $56^{\circ} \mathrm{C}$ for $1 \mathrm{~h}$. The digest was added to cultures at a concentration of $1 \mathrm{mg} / \mathrm{ml}$. A purified gliadin protein fraction was prepared and added to the cultures to obtain a concentration of $0.1 \mathrm{mg} / \mathrm{ml}$; this protein is an $\alpha$-gliadin fraction from "Scout 66" wheat and is called A-gliadin (12). Both PT and $\alpha$-gliadin exert a similar effect on tissue from patients with active GSE (1).

HLA typing. HLA-A and B locus antigens were determined by the National Cancer Institute Histocompatibility Typing Center, using a lymphocyte microcytotoxicity method previously described (13). Sera used to detect HLA antigens were obtained from the Serum Blood Bank maintained by the Transplantation and Immunology Branch of the National Institute of Allergy and Infectious Diseases, and from 
the Serum Collection of the Typing Center. The reproducibility of the assay when replicate determinations were done on normal individuals was $97 \%$. Normal HLA frequencies were determined by the study of random Caucasian blood bank donors. Presence or absence of HLA-D locus antigen, DRW3, was determined on eight patients using a previously described cytotoxicity assay (in conjunction with a specific antiserum for DRW3, kindly provided by Dr. J. J. Van Rood)(14).

Statistical analysis. The Wilcoxon rank-sum test was used to compare the base-line alkaline phosphatase activity in HLA-B8 positive and negative GSE groups. One-way analysis of variance was used to determine the significance of differences among all groups.

\section{RESULTS}

\section{HLA types}

$A$ and $B$ locus. 24 patients $(60 \%)$ with GSE were HLA-B8 positive and $16(40 \%)$ were HLA-B8 negative. The frequency of HLA-B8 in this group of patients with GSE was somewhat lower than that previously reported by us and others (3-5) because non-B8 patients were selected for study to equalize the size of the experimental groups. Such selections were made without prior knowledge of the patient's reactivity in organ culture.

DRW3 locus. Eight patients with GSE were studied for the presence of DRW3. Four of four HLA-B8 positive and three of four B8 negative patients were DRW3 positive. Thus, in this patient group, DRW3 has the same prevalence in B8 positive and B8 negative patients, as previously pointed out by Keunig et al. (6).

\section{Organ culture studies}

In previous studies, we showed that gluten sensitivity in vitro was demonstrable only in patients with active GSE and not in patients with inactive GSE. To make a meaningful comparison between HLA-B8 positive and B8 negative patients, it was, therefore, necessary to ensure that both groups of GSE patients had equivalent activity of disease. To this end, the HLA-B8 positive and B8 negative patients were compared with respect to intestinal alkaline phosphatase activity and jejunal morphology at the moment of biopsy. With regard to the former, we found that mean alkaline phosphatase values $( \pm S D)$ for $\mathrm{B} 8$ positive and $\mathrm{B} 8$ negative patients were $73 \pm 60$ and $112 \pm 90$, respectively. These values were statistically indistinguishable $(P>0.1)$ and are both lower than the mean enzyme value for normals in our laboratory $(350 \pm 75)$. With regard to jejunal morphology, we examined biopsies without foreknowledge of their source and found no significant difference between B8 positive and B8 negative patients: all biopsies had total or subtotal villus flattening and there was an equal amount of epithelial cell vacuolization and inflammation in the lamina propria of both groups. On the basis of these two objective features, it was concluded that B8 positive and B8 negative patients had equivalent levels of disease activity and could be expected to react to gluten in vitro.

\section{Comparison of $\mathrm{Z}$ values in organ cultures containing A-gliadin or PT digest}

A-gliadin or PT digest were used interchangeably. We compared the $Z$ values obtained in the groups using A-gliadin with the $Z$ values in the same groups using PT digest. This was done to assure that the results were not biased by the particular gluten preparation. Because the flat mucosa disease control group had only one A-gliadin value, that group was omitted from the analysis. The results of the comparison, using the Wilcoxon rank-sum test, showed there was no significant difference between $\mathrm{Z}$ values obtained with A-gliadin vs. PT digest $(P>0.05)$. This was in agreement with previous data (1). We, therefore, pooled all the data for each group in the subsequent analysis.

\section{GSE: HLA-B8 positive patients}

Biopsy specimens from 24 HLA-B8 positive patients were placed into organ culture. A-gliadin was present or absent in the cultures of 13 patients, while PT digest was used in cultures of the remaining 11 patients. Significant inhibition of alkaline phosphatase increase by gluten in organ culture, as indicated by a positive $\mathrm{Z}$ value, was observed in 11 of $13(85 \%)$ B8 positive patient cultures with A-gliadin and 10 of 11 (91\%) B8 positive patient cultures with PT digest (Table I). Thus, biopsy specimens from 21 of 24 (88\%) B8 positive patients demonstrated gluten-induced toxicity in vitro.

\section{HLA-B8 negative patients}

Biopsy specimens from 16 HLA-B8 negative patients were placed into organ culture. A-gliadin was present or absent in cultures of seven patients, while PT digest was present or absent in cultures of the remaining nine patients. Significant inhibition of alkaline phosphatase activity increases by gluten protein in organ culture, as indicated by positive $\mathrm{Z}$ values, was observed in only one of seven (14\%) cultures with A-gliadin and in only three of nine (33\%) cultures with PT digest (Table II). Thus, in contrast with the B8 positive patients, biopsy specimens from only 4 of $16(25 \%)$ B8 negative patients demonstrated gluten-induced toxicity in vitro. Except for the different response to gluten in vitro, HLA-B8 negative patients with GSE were indistinguishable in every respect from the patients who were HLA-B8 positive.

\section{Patients with abnormal small bowel biopsies not due to GSE}

Biopsy specimens from 15 patients with diarrhea and abnormal small bowel histology were placed into organ 
TABLE I

Alkaline Phosphatase Activity in Organ Culture: HLA-B8 Positive GSE Patients

\begin{tabular}{|c|c|c|c|c|}
\hline Patient No. & Base line & Without gluten & With gluten & Z Value* \\
\hline & & & A-gliadin & \\
\hline 1 & $90 \$$ & 149 & 52 & 2.3 \\
\hline 2 & 45 & 667 & 435 & -2.4 \\
\hline 3 & 47 & 157 & 97 & 1.1 \\
\hline 4 & 206 & 245 & 166 & 0.3 \\
\hline 5 & 55 & 238 & 166 & 0.1 \\
\hline 6 & 71 & 152 & 121 & 0.4 \\
\hline 7 & 250 & 626 & 317 & 0.5 \\
\hline 8 & 33 & 156 & 39 & 2.7 \\
\hline 9 & 82 & 128 & 80 & 1.2 \\
\hline 10 & 83 & 172 & 231 & -2.5 \\
\hline 11 & 76 & 210 & 136 & 0.6 \\
\hline 12 & 36 & 236 & 137 & 0.9 \\
\hline \multirow[t]{2}{*}{13} & 139 & 640 & 276 & 1.8 \\
\hline & & & PT digest§ & \\
\hline 14 & 135 & 458 & 191 & 2.0 \\
\hline 15 & 58 & 211 & 66 & 2.6 \\
\hline 16 & 9 & 63 & 26 & 2.0 \\
\hline 17 & 22 & 87 & 87 & 0.6 \\
\hline 18 & 0 & 86 & 46 & 1.7 \\
\hline 19 & 107 & 195 & 117 & 1.0 \\
\hline 20 & 47 & 155 & 40 & 2.7 \\
\hline 21 & 25 & 44 & 18 & 2.0 \\
\hline 22 & 94 & 180 & 50 & 2.7 \\
\hline 23 & 17 & 50 & 20 & 2.0 \\
\hline 24 & 43 & 100 & 125 & -0.4 \\
\hline
\end{tabular}

* $\mathrm{Z}=(0.0120)$ (without gluten value) $-(0.0284)$ (with gluten value $)+1.9975$. A positive value for $Z$ indicates in vitro gluten sensitivity.

\$ Units, $1 \mu \mathrm{mol}$ of $p$-nitrophenol phosphate hydrolyzed per gram tissue protein per minute. Each point is the mean of duplicate cultures determined in triplicate.

$\S$ PT digest of gluten (Frazer fraction III).

culture. A-gliadin was present in 1 and PT digest was present in 14 cultures. Significant inhibition of alkaline phosphatase activity increases by gluten protein in organ culture, as indicated by a positive $Z$ value, was observed in one patient with A-gliadin and one patient with PT digest (Table III). Thus, as previously noted (2), intestinal tissue which is abnormal because of causes other than GSE may, in a small fraction of cases, be subjected to nonspecific toxic injury by gluten in vitro.

\section{Patients with diarrhea and normal small bowel biopsy}

Biopsy specimens from 12 patients with diarrhea and normal small bowel histology were placed into organ culture: six with A-gliadin and six with PT digest
TABLE II

Alkaline Phosphatase Activity in Organ Culture: HLA-B8 Negative GSE Patients

\begin{tabular}{rrccc}
\hline Patient No. & Base line & Without gluten & With gluten & Z Value* \\
\hline & & & A-gliadin & \\
1 & $72 \ddagger$ & 414 & 476 & -6.6 \\
2 & 18 & 175 & 168 & -0.7 \\
3 & 20 & 115 & 171 & -1.5 \\
4 & 23 & 82 & 131 & -0.8 \\
5 & 190 & 400 & 490 & -7.1 \\
6 & 111 & 125 & 210 & -2.5 \\
7 & 304 & 459 & 250 & +0.4 \\
& & & & \\
& & & PT digest $\$$ & \\
8 & 304 & 459 & 305 & -1.1 \\
9 & 127 & 304 & 99 & +2.8 \\
10 & 61 & 342 & 138 & +2.2 \\
11 & 113 & 147 & 111 & +0.6 \\
12 & 10 & 211 & 159 & -0.01 \\
13 & 126 & 273 & 245 & -1.7 \\
14 & 146 & 145 & 280 & -4.2 \\
15 & 70 & 199 & 214 & -1.7 \\
16 & 98 & 222 & 253 & -2.5 \\
\hline
\end{tabular}

* $\mathrm{Z}=(0.0120)$ (without gluten value) $-(0.0284)$ (with gluten value) +1.9975 . A positive value for $\mathrm{Z}$ indicates in vitro gluten sensitivity.

I Units, $1 \mu \mathrm{mol}$ of $p$-nitrophenol phosphate hydrolyzed per gram tissue protein per minute. Each point is the mean of duplicate cultures determined in triplicate.

$\S$ PT digest of gluten (Frazer fraction III).

present in the culture medium. Significant inhibition of alkaline phosphatase activity increases by gluten protein in organ culture, as indicated by positive $\mathrm{Z}$ values, was not observed in any of these patients (Table IV).

\section{Normal individuals}

Biopsy specimens from eight normal subjects were placed into organ culture, three with A-gliadin and five with PT digest in the culture medium. Significant inhibition of alkaline phosphatase activity increases by gluten protein in organ culture, as indicated by positive $Z$ values, was not observed in any case (Table $V$ ). In summary, the correct classification rates in the GSE groups by A-gliadin and PT digest, respectively, were: $91 \%$ and $88 \%$ in the B8 positive group; and $14 \%$ and $33 \%$ in the B8 negative group (Fig. 1).

\section{DISCUSSION}

We previously showed that when evaluated prospectively, gluten sensitivity in vitro correlates with gluten sensitivity in vivo in most, but not all, instances. A 
TABLE III

Alkaline Phosphatase Activity in Organ Culture: Patients with Diarrhea and Flat Small Bowel Biopsy Not Due to GSE

\begin{tabular}{rrccc}
\hline Patient No. & Base line & Without gluten & With gluten & Z Value* \\
\hline & & & A-gliadin & \\
1 & $139 \ddagger$ & 338 & 205 & +0.2 \\
& & & PT digest $\$$ & \\
2 & 107 & 341 & 275 & -1.7 \\
3 & 82 & 90 & 118 & -0.3 \\
4 & 19 & 91 & 126 & -0.5 \\
5 & 74 & 85 & 124 & -0.5 \\
6 & 58 & 118 & 94 & +0.7 \\
7 & 42 & 45 & 410 & -10 \\
8 & 32 & 310 & 285 & -2.4 \\
9 & 83 & 106 & 134 & -0.5 \\
10 & 175 & 361 & 317 & -2.7 \\
11 & 16 & 250 & 300 & -3.5 \\
12 & 69 & 236 & 256 & -2.4 \\
13 & 25 & 317 & 285 & -2.3 \\
14 & 143 & 242 & 207 & -1 \\
15 & 83 & 230 & 207 & -1.1 \\
\hline
\end{tabular}

* $\mathrm{Z}=(0.0120)$ (without gluten value) - (0.0284) (with gluten value $)+1.9975$. A positive value for $Z$ indicates in vitro gluten sensitivity.

† Units, $1 \mu \mathrm{mol}$ of $p$-nitrophenol phosphate hydrolyzed per gram tissue protein per minute. Each point is the mean of duplicate cultures determined in triplicate.

$\S$ PT digest of gluten (Frazer fraction III).

possible explanation for the lack of complete correlation is provided by the data in the current study. Although by standard clinical, chemical, and intestinal morphologic criteria, no evident differences exist between patients with GSE who are HLA-B8 positive or negative, a clear difference exists with regard to sensitivity to gluten in vitro between HLA-B8 positive and negative patients. Thus, intestinal biopsies of HLA-B8 positive patients show a consistent sensitivity to gluten when tested in vitro. On the other hand, intestinal biopsies from patients who are HLA-B8 negative and clearly have gluten sensitivity in vivo, are indistinguishable from biopsies of controls, each showing no consistent sensitivity to gluten in vitro. Therefore, if used to assess the diagnosis of GSE prospectively, the usefulness of the in vitro technique can be enhanced by taking the patients' histocompatibility antigen into account.

One possible explanation for the difference between the effect of gluten in vivo and in vitro is that gluten presented to the intestinal mucosa in vivo differs from gluten presented in vitro. In fact, gluten protein is subjected to nearly complete proteolytic degradation in vivo, whereas proteolytic degradation in vitro (used to
TABLE IV

Alkaline Phosphatase Activity in Organ Culture: Patients with Diarrhea and Normal Small Bowel Biopsy

\begin{tabular}{|c|c|c|c|c|}
\hline Patient No. & Base line & Without gluten & With gluten & Z Value ${ }^{*}$ \\
\hline & & & A-gliadin & \\
\hline 1 & $378 \ddagger$ & 674 & 907 & -15.7 \\
\hline 2 & 139 & 196 & 242 & -2.5 \\
\hline 3 & 242 & 365 & 381 & -4.4 \\
\hline 4 & 173 & 322 & 291 & -2.4 \\
\hline 5 & 329 & 498 & 509 & -6.5 \\
\hline \multirow[t]{2}{*}{6} & 270 & 279 & 511 & -9.2 \\
\hline & & & $\underline{\text { PT digest } \$}$ & \\
\hline 7 & 236 & 435 & 470 & -6.1 \\
\hline 8 & 464 & 683 & 560 & -5.7 \\
\hline 9 & 205 & 310 & 296 & -2.7 \\
\hline 10 & 322 & 462 & 445 & -5.1 \\
\hline 11 & 284 & 627 & 682 & -9.9 \\
\hline 12 & 306 & 404 & 566 & -9.2 \\
\hline
\end{tabular}

$\ddagger$ Units, $1 \mu \mathrm{mol}$ of $p$-nitrophenol phosphate hydrolyzed per gram tissue protein per minute. Each point is the mean of duplicate cultures determined in triplicate.

$\S$ PT digest of gluten (Frazer fraction III).

prepare Frazer fraction III) is likely to be only partial. On this basis, it is possible that B8 positive GSE tissue and B8 negative GSE tissue differ in their susceptibility

TABLE V

Alkaline Phosphatase Activity in Organ Culture: Normal Individuals

\begin{tabular}{ccccc}
\hline Patient No. & Base line & Without gluten & With gluten & Z Value* \\
\hline & & & A-gliadin & \\
1 & $345 \ddagger$ & 668 & 628 & -7.8 \\
2 & 153 & 187 & 205 & -1.6 \\
3 & 173 & 322 & 291 & -2.4 \\
& & & PT digest $\$$ & \\
4 & 353 & 467 & 480 & -5.8 \\
5 & 236 & 465 & 470 & -6.2 \\
6 & 321 & 590 & 590 & -7.7 \\
7 & 221 & 550 & 600 & -8.4 \\
8 & 395 & 515 & 600 & -8.8 \\
\hline
\end{tabular}

* $\mathrm{Z}=(0.0120)$ (without gluten value) $-(0.0284)$ (with gluten value) +1.9975 . A positive value for $\mathrm{Z}$ indicates in vitro gluten sensitivity.

† Units, $1 \mu \mathrm{mol}$ of $p$-nitrophenol phosphate hydrolyzed per gram tissue protein per minute. Each point is the mean of duplicate cultures determined in triplicate.

$\S$ PT digest of gluten (Frazer fraction III). 


\section{$Z$ VALUES IN PATIENTS WITH GSE AND CONTROLS}

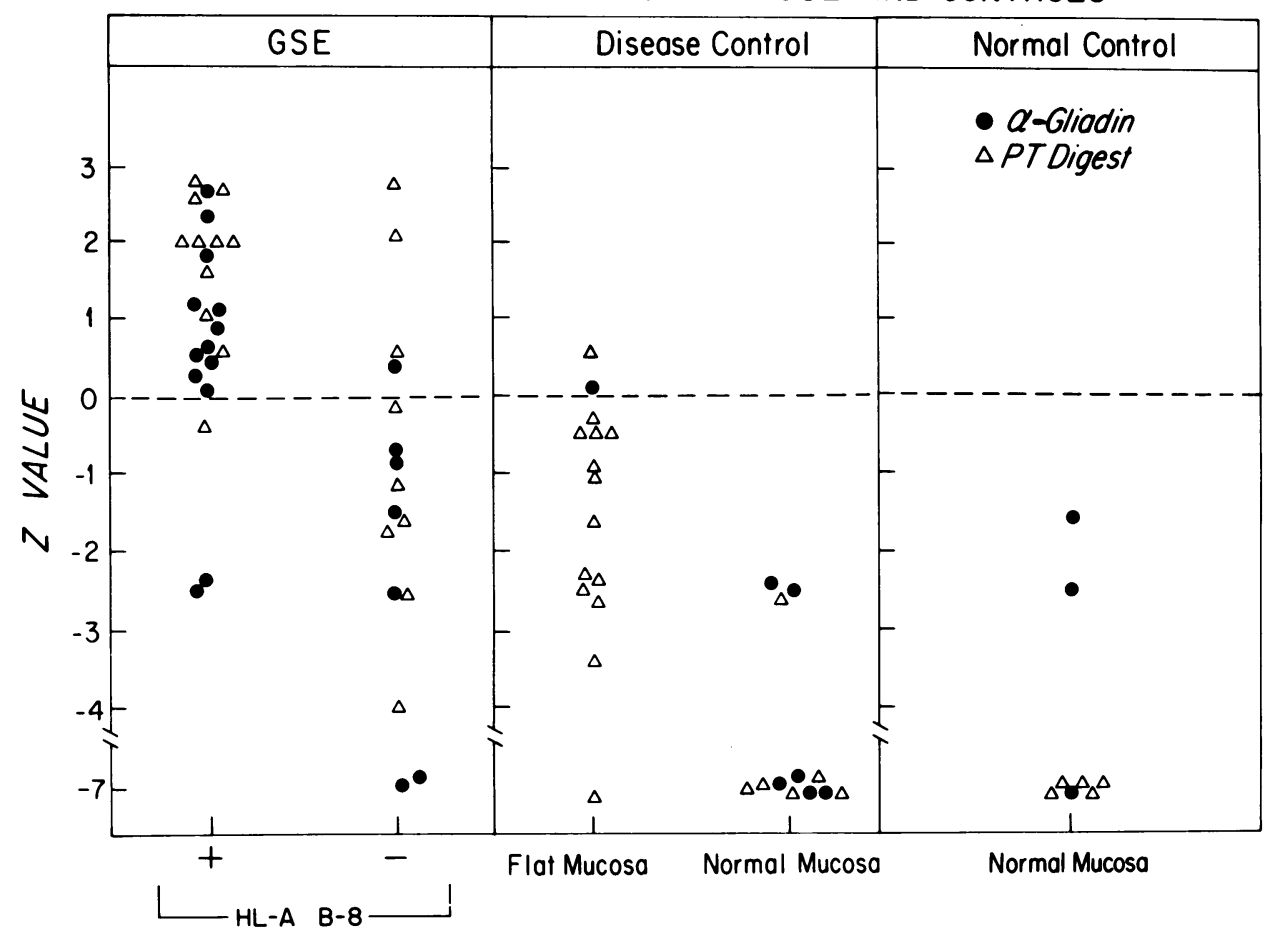

FIGURE 1 Z values in HLA-B8 positive and negative GSE patients, diseased, and normal controls. The values for $Z$ are negative in all normals, in 13 of 15 diseased controls, and 12 of 16 HLA-B8 negative patients with GSE. In contrast, the $\mathrm{Z}$ value is positive in 21 of $24 \mathrm{HLA}$-B8 positive patients with GSE. The difference in distribution in the $\mathrm{Z}$ values is significant at $P<0.01$ (five group one-way analysis of variance).

to damage by intact gluten protein vs. partial degradation products of gluten. This difference in susceptibility to the various molecular forms of gluten will be revealed only in vitro, where such chemical forms can be studied.

It has been postulated that gluten injures the intestine of susceptible persons by stimulating an immune response characterized by mucosal antigluten antibody synthesis (15) and production of sensitized T cells (16). Rubin et al. (17) indicated that gluten binds to the epithelial cell of patients with GSE. We suggest that the various intestinal immune reactions occur as a consequence of gluten binding to the epithelial cell; i.e., unbound gluten is not immunogenic, whereas bound gluten behaves as a hapten.

A possible mechanism which could account for the influence of histocompatibility antigen on tissue cytotoxicity is that the HLA gene product, itself, forms part of the target of cytotoxicity. The effector cell recognizes the target of cytotoxicity either as a complex target composed of a closely associated, but separate modifying antigen (gluten), and the histocompatibility antigen (dual recognition), or recognizes the single modified histocompatibility antigen (gluten plus histocompatibility antigen) (modified self). There is experimental support for these possibilities in other experimental systems (18). Using this concept in GSE, since the histocompatibility antigen itself forms a part of the target of cytotoxicity, HLA-B8 positive and negative patients could be expected to show different degrees of toxicity to gluten in vitro.

The precise mechanism of the cytotoxic event in GSE remains unidentified. Douglas (19) and Weiser et al. (20) have postulated that the binding of gluten, itself, is directly toxic. We and others have theorized that the relevant cytotoxic mechanism in GSE is either a $T$ cell-mediated mechanism or a B cell-mediated mechanism. In the former instance, histocompatibility antigens facilitate binding of gluten or gluten fragment to epithelial cells and, in addition, form part of the target on epithelial cells recognized by cytotoxic $T$ cells. In the latter instance, histocompatibility antigens facilitate binding of gluten or gluten fragment to epithelial cells, but do not form part of the epithelial target recognized by cytotoxic antibodies or antibodies cooperating with $\mathrm{K}$ cells - only the gluten would be involved. (The $\mathrm{K}$ cell, also known as the null lymphocyte, mediates antibody-dependent, cell-mediated cytotoxicity.) Use of the in vitro model may further answer the question. 


\section{ACKNOWLEDGMENTS}

Dr. Falchuk is supported in part by National Institutes of Health grant AM17684 and Research Career Development Award AM 00210. Dr. Katz is a recipient of a Basil O'Connor Starter Award and Clinical Investigator Award AM 00234 from the National Institutes of Health.

\section{REFERENCES}

1. Falchuk, Z. M., R. L. Gebhard, C. Sessoms, and W. Strober. 1974. An in vitro model of gluten-sensitive enteropathy. J. Clin. Invest. 53: 487-500.

2. Katz, A. J., and Z. M. Falchuk. 1978. Definitive diagnosis of gluten-sensitive enteropathy. Use of an in vitro organ culture model. Gastroenterology. 75: 695-700.

3. Falchuk, Z. M., G. N. Rogentine, and W. Strober. 1972. Predominance of histocompatibility antigen HLA-8 in patients with gluten-sensitive enteropathy.J. Clin. Invest. 51: 1602-1605.

4. Stokes, P. L., P. Asquith, G. K. T. Holmes, P. Macintosh, and W. T. Cook. 1972. Histocompatibility antigen associated with adult coeliac disease. Lancet. II: 162-164.

5. Harms, K., G. Granditsch, E. Rossipal, H. Ludwig, Z. Polymenidis, S. Scholz, R. Wank, and E. D. Albert. 1974. HLA in patients with coeliac disease and their families. In Coeliac Disease, W. Th. J. M. Hekkens and A. S. Peña, editors. Stenfert Kroese, Leiden. 215-220.

6. Keuning, J. J., A. S. Peña, A. van Leeuwen, J. P. van Hoof, and J. J. van Rood. 1976. HLA-DW3 association with coeliac disease. Lancet. I: 506-508.

7. BMDP Biomedical Computer Programs, P-series. 1977. W. J. Dixon and M. B. Brown, editors. University of California Press, Berkeley. Program P7M. 711-733.

8. Brandborg, L. L., C. E. Rubin, and W. E. Quinton. 1959. A multipurpose instrument for suction biopsy of the esophagus, stomach, small bowel, and colon. Gastroenterology. 37: 1-16.

9. Lowry, O. H., N. J. Rosebrough, A. L. Farr, and R. J. Randall. 1951. Protein measurement with the Folin phenol reagent. J. Biol. Chem. 193: 265-275.
10. Bessey, O. A., O. H. Lowry, and M. J. Brock. 1946. A method for the rapid determination of alkaline phosphatase with five cubic millimeters of serum. J. Biol. Chem. 164: 321-329.

11. Frazer, A. C., R. F. Fletcher, C. A. C. Ross, B. Shaw, H. G. Sammons, and R. Schneider. 1959. Gluten-induced enteropathy: the effect of partially digested gluten. Lancet. II: $252-255$.

12. Kasarda, D. D., C. C. Nimmo, and J. E. Bernardin. 1974. Structural aspects and genetic relationships of gliadin. In Coeliac Disease. Proceedings 2nd International Symposium. W. Th. J. M. Hekkens and A. S. Peña, editors. Stenfert Kroese, Leiden. 25-36.

13. Mittal, K. K., M. R. Mickey, O. D. Singal, and P. I. Terasaki. 1968. Serotyping for homotransplantation. XVIII. Refinement of microdroplet lymphocyte cytotoxicity test. Transplantation (Baltimore). 6: 913-920.

14. Mann, D. L., S. I. Katz, D. L. Nelson, L. D. Abelson, and W. Strober. 1976. Specific B-cell antigens associated with gluten-sensitive enteropathy and dermatitis herpetiformis. Lancet. I: 110-111.

15. Falchuk, Z. M., and W. Strober. 1974. Gluten-sensitive enteropathy: synthesis of antigliadin antibody in vitro. Gut. 15: 947-952.

16. Ferguson, A., T. T. MacDonald, J. P. McClure, and R. J. Hadden. 1975. Cell-mediated immunity to gliadin within the small intestinal mucosa in coeliac disease. Lancet. I: $895-897$.

17. Rubin, W., A. S. Fauci, M. H. Sleisenger, and G. H. Jeffries. 1965. Immunofluorescent studies in adult celiac disease. J. Clin. Invest. 44: 475-485.

18. Shearer, G. M., and A. M. Schmitt-Verhulst. 1977. Major histocompatibility complex restricted cell-mediated immunity. Adv. Immunol. 25: 55-87.

19. Douglas, A. P. 1976. The binding of glycopeptide component of wheat to intestinal mucosa of normal and coeliac human subjects. Clin. Chim. Acta. 73: 357-360.

20. Weiser, M., and A. P. Douglas. 1976. An alternative mechanism for gluten toxicity in coeliac disease. Lancet. I: 567-569. 